\title{
Un-functionalized Gold Nanoparticles as a Simple Colorimetric Probe for Sensitive and Selective Detection of Dopamine
}

\author{
Nokuthula Khanyile $^{\mathrm{a}, *(\mathbb{D}) \S}$, Rui Krause ${ }^{\mathrm{b}}$, Sibulelo Vilakazi ${ }^{\mathrm{c}}$ and Nelson Torto $^{\mathrm{d}}$ \\ ${ }^{a}$ School of Chemical and Physical Science, University of Mpumalanga, Private Bag X11283, Mbombela, South Africa. \\ ${ }^{b}$ Chemistry Department, Rhodes University, P.O. Box 94, Grahamstown, South Africa. \\ 'Advanced Materials Division, Nanoscience and Nanotechnology, 200 Malibongwe Drive 2125, Randburg, South Africa. \\ ${ }^{d}$ Botswana Institute for Technology Research and Innovation, Private Bag 0082, Gaborone, Botswana.
}

Received 21 June 2018, revised 24 May 2019, accepted 14 June 2019.

\begin{abstract}
A dopamine (DA) colorimetric probe based on the growth and aggregation of un-functionalized gold nanoparticles (AuNPs) is reported. Upon addition of AuNPs to dopamine at various concentrations, the shape, size and colour change of the nanoparticles results in spectral shifts to higher wavelengths and hence colour change is the mode of detection. The colour change can be easily observed by the naked eye from as low as $5.0 \mathrm{nM}$ DA, even under sub-optimal conditions. Under optimal $\mathrm{pH}$ conditions the calculated limit of detection was $2.5 \mathrm{nM}(3 \sigma)$. The probe was successfully applied to whole blood sample and showed good selectivity and sensitivity towards DA. The simple, sensitive and selective probe could be an excellent alternative for on-site and immediate detection of DA without the use of instrumentation and would thus be useful for rapid diagnostic applications.
\end{abstract}

KEYWORDS

Dopamine, gold nanoparticles, colorimetric probe, neurotransmitter, sensor.

\section{Introduction}

Dopamine (DA) is an important neurotransmitter that plays a number of physiological roles in the mammalian central nervous system, human metabolism, cardiovascular, renal, and hormonal systems..$^{1-5}$ It is formed by decarboxylation of L-3,4dihydroxyphenylaniline ${ }^{6}$ which is also known as L-DOPA. Dopamine affects the fundamental functions of the brain such as reward-related behaviour, movement and mood. ${ }^{7}$ Current methods for the accurate detection of DA, including spectroscopic and electrochemical, are time consuming, often lack selectivity and require technically competent personnel and equipment. ${ }^{8}$ Moreover, DA, ascorbic acid (AA) and uric acid (UA) are found together in biological samples. ${ }^{1} \mathrm{AA}$ and UA usually cause interferences during DA determination. There is therefore a considerable demand for an analytical method that is not only sensitive, simple, and rapid but also has good selectivity for DA detection (especially over AA and UA).

Methods to detect biological and inorganic compounds have been reported for many years. Newly developed methods are usually characterized by advantages over conventionally accepted methods, particularly in sensitivity and selectivity. ${ }^{9-12}$ Among the newer methods, colorimetric detection appears to be commonly applied to routine analysis because it is simple, cheap and the detection can be achieved with the naked eye, ${ }^{13}$ thus removing the need for complicated instruments. There are reports on colorimetric detection of DA. However, they are limited due to low selectivity and sensitivity or are associated with complex procedures. ${ }^{14}$ To overcome the challenges in detecting DA, in this study, gold nanoparticles (AuNPs) were employed.

* To whom correspondence should be addressed.

E-mail: nokuthulangomane24@gmail.com
AuNPs have attracted great interest as components of colorimetric probes owing to properties such as stability, biocompatibility, ${ }^{15}$ ease of preparation and distinct spectral properties. ${ }^{16}$ AuNPs are known to have colours that range from red to purple and blue depending on the formation of aggregates, size and shape of the particles, which results in changes that can easily be perceived with the naked eye. AuNP-based colorimetric probes are exceptionally sensitive due to their high extinction coefficients compared with traditional organic chromophores. ${ }^{17}$ Selective colorimetric detection of DA based on functionalized AuNPs has been explored and reported. For example, dithiobis(succinimidylpropionate), ${ }^{7,18}$ 4-mercaptophenylboronic and 4-(dimethylamino)pyridine ${ }^{19}$ functionalized AuNPs were applied as probes for optical detection of DA. The compounds employed to functionalize the AuNPs were used as stabilizers of the AuNPs and also for enhancing the selectivity of the probes towards DA. ${ }^{7}$ These methods all rely on consistent functionalization and are therefore sensitive to variations in the manufacture and storage conditions. Herein, we report a technique to detect DA employing un-functionalized AuNPs in aqueous samples. The selectivity and sensitivity of the method was evaluated in whole blood samples. In this study, un-functionalized AuNPs are used for the first time to detect DA.

\section{Experimental}

\subsection{Reagents and Materials}

Reagents and materials used in this study are similar to those that were used in our previous publication. ${ }^{20}$ Gold (III) chloride hydrate $(99.999 \%)\left(\mathrm{HAuCl}_{4} \cdot \mathrm{xH}_{2} \mathrm{O}\right)$, sodium borohydride $(98.0 \%)\left(\mathrm{NaBH}_{4}\right)$, sodium acetate tri-hydrate $\left(\mathrm{C}_{2} \mathrm{H}_{9} \mathrm{NaO}_{5}\right)$, dopamine hydrochloride $(99.95 \%)\left(\mathrm{DA}, \mathrm{C}_{8} \mathrm{H}_{11} \mathrm{NO}_{2} \mathrm{D} \cdot \mathrm{HCl}\right)$, uric acid $(99.0 \%)\left(\mathrm{UA}, \mathrm{C}_{5} \mathrm{H}_{4} \mathrm{~N}_{4} \mathrm{O}_{3}\right)$ and ascorbic acid $(99.0 \%)\left(\mathrm{AA}, \mathrm{C}_{6} \mathrm{H}_{8} \mathrm{O}_{6}\right)$ 
were purchased from Sigma-Aldrich (Saint Louis, MO, USA). Acetic acid $(99.5 \%)\left(\mathrm{CH}_{3} \mathrm{CO}_{2} \mathrm{H}\right)$, hydrochloric acid $(32.0 \%)(\mathrm{HCl})$ and nitric acid $(55.0 \%)\left(\mathrm{HNO}_{3}\right)$ were from B \& M Scientific CC (Western Cape, South Africa). Formic acid $(85.0 \%)\left(\mathrm{HCO}_{2} \mathrm{H}\right)$ was purchased from Minema (Gauteng, South Africa). Sodium dihydrogen phosphate $\left(\mathrm{NaH}_{2} \mathrm{PO}_{4}\right)$ was purchased from May \& Baker (Dagenham, England) and sodium hydroxide $(\mathrm{NaOH})$ was from Merck (Gauteng, South Africa). All chemicals were of analytical grade and used as received. All the glassware and magnetic stirrer bar used were washed with freshly prepared aqua regia $\left(1: 3 \mathrm{HNO}_{3}: \mathrm{HCl}\right)$ and rinsed thoroughly with deionized water before use. Absorbance measurements were performed using a Lambda 25 Perkin-Elmer UV spectrophotometer (Santa Clara, CA, USA). Ultrapure water obtained from a Millipore RiOS ${ }^{\mathrm{TM}} 16$ and Milli-Q Academic ${ }^{\circledast}$ A10 system (Milford, MA, USA) was used throughout this study.

\subsection{Preparation of the Un-functionalized AuNPs Probe}

The un-functionalized gold nanoparticles (UF-AuNPs) were synthesized by dissolving $39 \mathrm{mg}$ of $\mathrm{HAuCl}_{4} \cdot \mathrm{xH}_{2} \mathrm{O}$ in $10 \mathrm{~mL}$ of water. $\mathrm{NaBH}_{4}(0.3 \mathrm{mg})$ was added in solid form while the solution was being stirred vigorously. The solution was constantly stirred for $30 \mathrm{~min}$ at room temperature.

\subsection{Procedure of Colorimetric Detection of DA}

Various concentrations of DA $(0.005 \mathrm{mM}-2.5 \mathrm{M})$ were prepared and $5 \mathrm{~mL}$ of each concentration used for the determination. The AuNPs solution $(100 \mu \mathrm{L})$ was added to the $5 \mathrm{~mL}$ DA solutions. The resulting mixture was transferred to a $1 \mathrm{~cm}$ pathlength quartz cuvette to record the absorption spectra over the wavelength range of 270 to $700 \mathrm{~nm}$.

\subsection{Selectivity Studies}

For selectivity studies, AA and UA (each $0.125 \mathrm{M}), \mathrm{DA}(0.05 \mathrm{M})$ and mixtures of AA, UA and DA were prepared. Then the procedure in section 2.3 (addition of AuNPs) was followed to investigate the selectivity of the probe towards DA.

\section{5. $\mathrm{pH}$ Studies}

For the $\mathrm{pH}$ studies, two types of buffer solutions (acetate and phosphate) were used to adjust the $\mathrm{pH}$ of the solutions. The acetate buffer was prepared by mixing $10 \mathrm{mM}$ of sodium acetate tri-hydrate and $10 \mathrm{mM}$ acetic acid, and this was used for $\mathrm{pH} 2-6$, whilst the phosphate buffer prepared by mixing $10 \mathrm{mM}$ sodium dihydrogen phosphate and $10 \mathrm{mM}$ of sodium hydroxide was used for $\mathrm{pH} 7-11$. Dopamine $(5 \mu \mathrm{M})$ was used as a representative for the study and the profile of the absorbance ratio $\left(\mathrm{A}_{388} / \mathrm{A}_{308}\right)$ was plotted against $\mathrm{pH}$.

\subsection{Detection of DA Under Controlled Conditions}

Firstly, $1 \mathrm{~mL}$ of the buffer solution (phosphate buffer $\mathrm{pH} 8$ ) was added to $50 \mu \mathrm{L}$ of the UF-AuNPs. Then $4 \mathrm{~mL}$ of various concentrations of DA solutions $(5 \mathrm{nM}-50 \mathrm{mM})$ were added to the mixture. The solutions were incubated at room temperature $\left(25 \pm 2{ }^{\circ} \mathrm{C}\right)$ for $2 \mathrm{~min}$ before the absorbance was recorded at 308 and $388 \mathrm{~nm}$. Five absorbance readings were taken for each concentration.

\subsection{Colorimetric Detection of DA in Whole Blood Sample}

Whole blood samples were purchased from Seronorm (Billingstad, Norway). The sample was diluted 100 times with phosphate buffer ( $\mathrm{pH}$ 8) before analysis, and no other pre-treatment was required. Particularly, $3 \mathrm{~mL}$ of $5 \mu \mathrm{M}$ and $50 \mu \mathrm{M}$ DA was added to $2 \mathrm{~mL}$ of the sample solution and subse- quently, $50 \mu \mathrm{L}$ of the UF-AuNPs was added. Absorption spectra were recorded after incubation at room temperature as before, for $2 \mathrm{~min}$.

\section{Results and Discussion}

\subsection{Visual and Spectroscopic Characteristics of DA}

The $\mathrm{NaBH}_{4}$ reduced UF-AuNPs were green in colour and were stable against aggregation for more than 3 months (stored in a cupboard or in the refrigerator at $4{ }^{\circ} \mathrm{C}$ in the dark). The stability was attributed to excess $\mathrm{AuCl}_{4}^{-}$and $\mathrm{AuCl}_{2}^{-}$ions that bind to the surface of the gold nanoparticles in the solution during the reduction of $\mathrm{AuCl}_{4} \cdot{ }^{-16} \mathrm{Fig}$. 1A shows the colours of the solutions of different concentrations of DA that resulted from reacting with $100 \mu \mathrm{L}$ of the AuNPs. The $0 \mathrm{M}$ solution (pale green) was used as a control. Colour changes from pale green to pale orange $(0.5 \mathrm{mM})$, orange $(5 \mathrm{mM})$, dark orange $(0.05 \mathrm{M})$, wine-red $(0.5$ and $1 \mathrm{M})$ and brown $(2.5 \mathrm{M})$ were observed immediately after addition of various concentrations of DA. The series of photographs and corresponding absorption spectra are shown in Fig. 1A and Fig. 1B, respectively. Additional DA concentrations of $1.25 \mathrm{mM}, 2.50 \mathrm{mM}$, , $12.5 \mathrm{mM}, 25.0 \mathrm{mM}, 0.125 \mathrm{M}, 0.250 \mathrm{M}$ and $0.625 \mathrm{M}$ were also prepared in order to better evaluate the subtle spectroscopic changes (Fig. 1B). The absorption band of the gold salt before addition of any $\mathrm{NaBH}_{4}$ had an observed maximum at $294 \mathrm{~nm}$ as shown in Fig. 1B (a1).

$\mathrm{AuCl}_{4}^{-}$ions have been reported to show an absorption band at $220 \mathrm{~nm}$ with a shoulder at $290 \mathrm{~nm}$; this is caused by charge transfer between gold and the chloro ligands, ${ }^{21}$ which was also observed in this case. After reduction with $\mathrm{NaBH}_{4}$, but before addition of the analyte (DA), the absorption band had shifted to $308 \mathrm{~nm}$ (Fig. 1B, a). The band at $308 \mathrm{~nm}$ confirms that nanoparticles were formed but the borohydride that was added could not reduce all the $\mathrm{AuCl}_{4}^{-}$ions. Absorbance decreased progressively at $308 \mathrm{~nm}$ with increasing concentration of DA was observed, especially at very low DA concentrations of $0.005 \mathrm{mM}$ and $0.05 \mathrm{mM}$ (Fig. 1B, a-c). These spectral shifts are not sufficient to allow visual detection of DA, but may be developed in a spectrophotometric method. On addition of significant amounts of DA (1.25 mM, 2.5 mM, 0.0125 M and $0.025 \mathrm{M})$ an absorption maximum at $388 \mathrm{~nm}$ was observed (Fig. 1B, d-g). A second band at $500 \mathrm{~nm}$ was observed for higher DA concentrations of 0.125 , $0.25,0.625,1$ and $2.5 \mathrm{M}$ (Fig. $1 \mathrm{~B}, \mathrm{~h}-\mathrm{j}$ ). Dopamine is known to act as a good reducing agent in certain circumstances. ${ }^{22}$ When the green AuNPs were mixed with the different concentrations of $\mathrm{DA}$, more and more of the remaining $\mathrm{AuCl}_{4}^{-}$and $\mathrm{AuCl}_{2}^{-}$ions are reduced to $\mathrm{Au}^{0}$ nanoparticles, thus resulting in the reduction of the initial band at $308 \mathrm{~nm}$. The newly formed nanoparticles attached themselves onto the existing nanoparticles to form bigger nanoparticles or aggregate particles resulting in colour changes and shifts in the absorption spectra from lower to higher wavelengths (Scheme 1). A similar process is common in the deliberate synthesis of shaped nanoparticles from small 'seed' nanoparticles. ${ }^{23}$

In order to understand how the spectral features of the probe are related to particle size and shape, transmission electron microscopy (TEM) studies were conducted. The TEM images (Fig. 2) were in agreement with the UV-visible spectroscopy results as they showed an increase in the size of the nanoparticles and that aggregates were formed. The synthesized nanoparticles were initially well dispersed, roughly spherical in shape and their average diameter was estimated to be $10 \mathrm{~nm}$ (Fig. 2a). The TEM images confirmed growth and aggregation of the AuNPs in the presence of DA (Fig. 2, b-f). Interestingly, TEM 

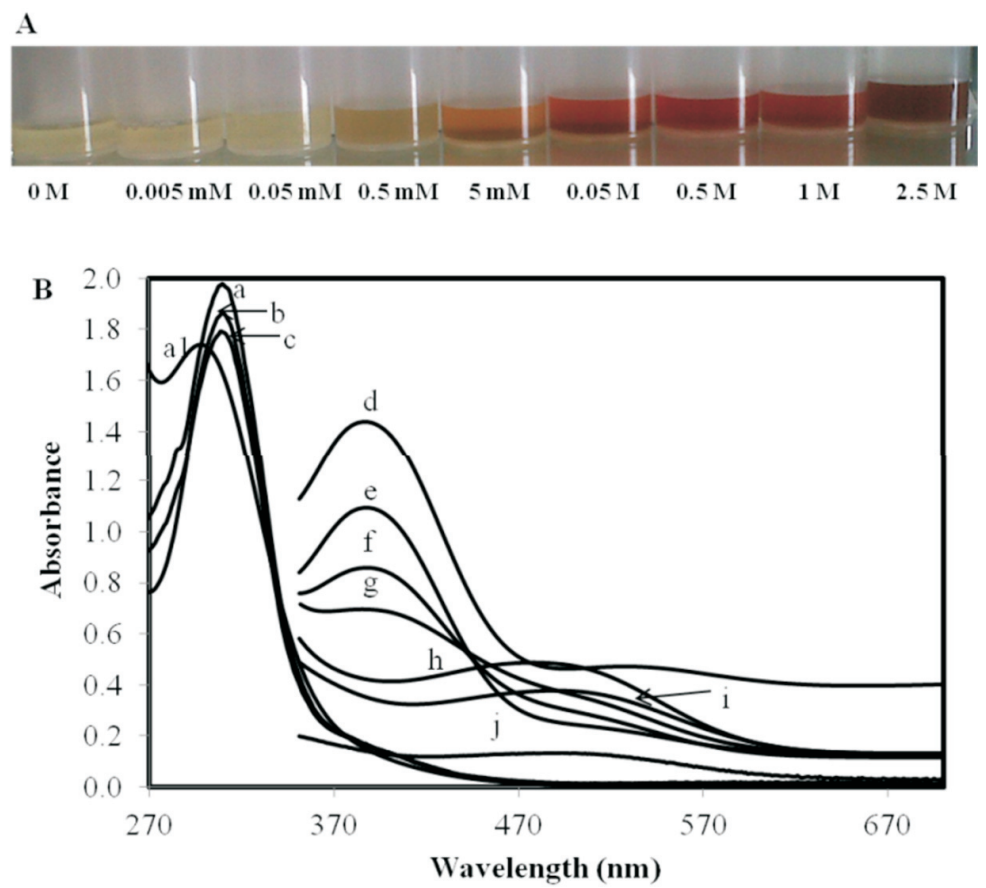

Figure 1 (A) Photograph showing colour changes of solutions of different concentrations of DA reacted with $100 \mu \mathrm{L}$ of the UF-AuNPs; (B) absorbance spectra of: a, Au salt before $\mathrm{NaBH}_{4}$, AuNPs in DA solutions a, 0 M; b, $0.005 \mathrm{mM}$; c, $0.05 \mathrm{mM}$; d, $1.25 \mathrm{mM}$; e, 2.5 mM; f, 0.0125 M; g, 0.025 M; h, 0.125 M; i, $0.625 \mathrm{M} ; \mathrm{j}, 2.5 \mathrm{M}$.

also revealed the generation of different shapes of the AuNPs for different concentrations of DA. Again this is consistent with a 'seed' approach to AuNP synthesis. ${ }^{23}$

\subsection{Selectivity}

The selectivity of the UF-AuNPs for DA detection in the presence of interferences (ascorbic acid and uric acid) was investigated. The results shown in Fig. 3 demonstrate that there was no colour change when the UF-AuNPs were introduced to a relatively high concentration of UA (Fig. $3 \mathrm{~A}, \mathrm{~b}$ ) and there was also no significant spectral change for this solution (Fig. 3B, b). An orange colour was observed for DA (Fig. 3A, c) while the colour changed to purple in AA (Fig. 3A, d) with absorption maxima around 498 and $609 \mathrm{~nm}$, respectively (Fig, 3B, c and d). The response of AA to the nanoparticle probe was not surprising since it is known that AA and DA have similar chemical properties. In this case, both chemicals have been used as reducing agents for nanoparticle synthesis. ${ }^{24,25}$ In this study, however, while it is likely that AA like DA is reducing excess $\mathrm{AuCl}_{4}^{-}$and $\mathrm{AuCl}_{2}^{-}$ions, the resulting nanoparticles are different and present a very different colour. Interestingly, a mixture of the three analytes resulted in an orange colour (Fig, 3A, e) with absorption maxima at $490 \mathrm{~nm}$ (Fig. 3B, e) corresponding to that of DA yet the concentration of DA was much lower (almost one third of both $\mathrm{AU}$ and $\mathrm{AA})$.

Based on the results in Fig. 3, the UF-AuNPs probe can be used to detect $\mathrm{AA}$ in the absence of the interfering compounds. AA was further investigated as a possible interfering species because of the observed colour change. Different concentrations of AA were prepared to investigate the extent to which the probe could detect AA. Colour changes were observed from light green to purple for $0.125 \mathrm{mM}$ to $1 \mathrm{M} \mathrm{AA}$ (Fig. 4A).

The absorption maxima for the nanoparticles in the presence of AA were between 553 and $613 \mathrm{~nm}$ (Fig. 4B, c-g) while the ones for the reduced particles alone was at $308 \mathrm{~nm}$ (Fig. 4B, a and b).

Next, concentrations of DA and AA were varied and mixed to investigate if the probe was selective to various concentrations of DA in the existence of AA. The results are shown in Fig. 5. An orange colour was observed on addition of the AuNPs to a solution of DA, even when AA concentrations were 20 times greater or 50 times lower than that of DA (Fig. 5A, b and c, respectively). The corresponding absorption spectra $\left(\lambda_{\max }\right.$ around $\left.500 \mathrm{~nm}\right)$ (Fig. 5B, b) were similar to those that were observed for DA in the absence of AA. When the concentration of AA was increased to 200 times greater than that of DA, a purple colour was observed (Fig. $5 \mathrm{~A}, \mathrm{a})$ and the corresponding absorption spectra $\left(\lambda_{\max }\right.$ around $605 \mathrm{~nm}$ ) was similar to that observed for AA in the absence of DA (Fig. 5B, a). It is clear that the probe can be used for detecting both AA and DA, separately, or for detecting DA in the presence of moderate amounts of AA. In biological samples where concentrations of AA are higher than those of DA (usually 100-1000 times higher) the probe will only remain effective until

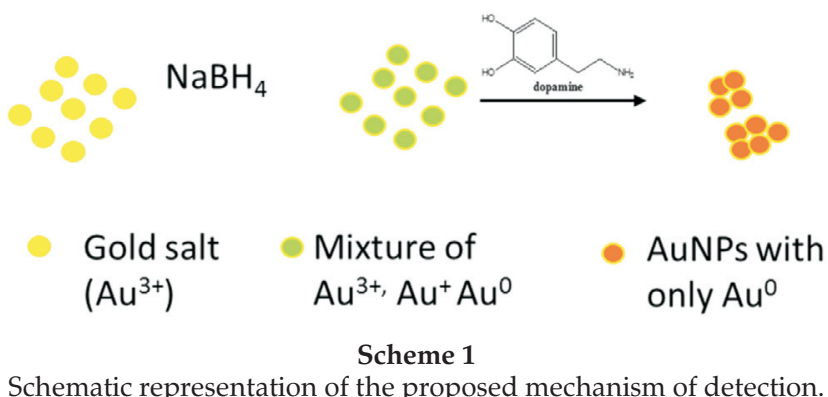



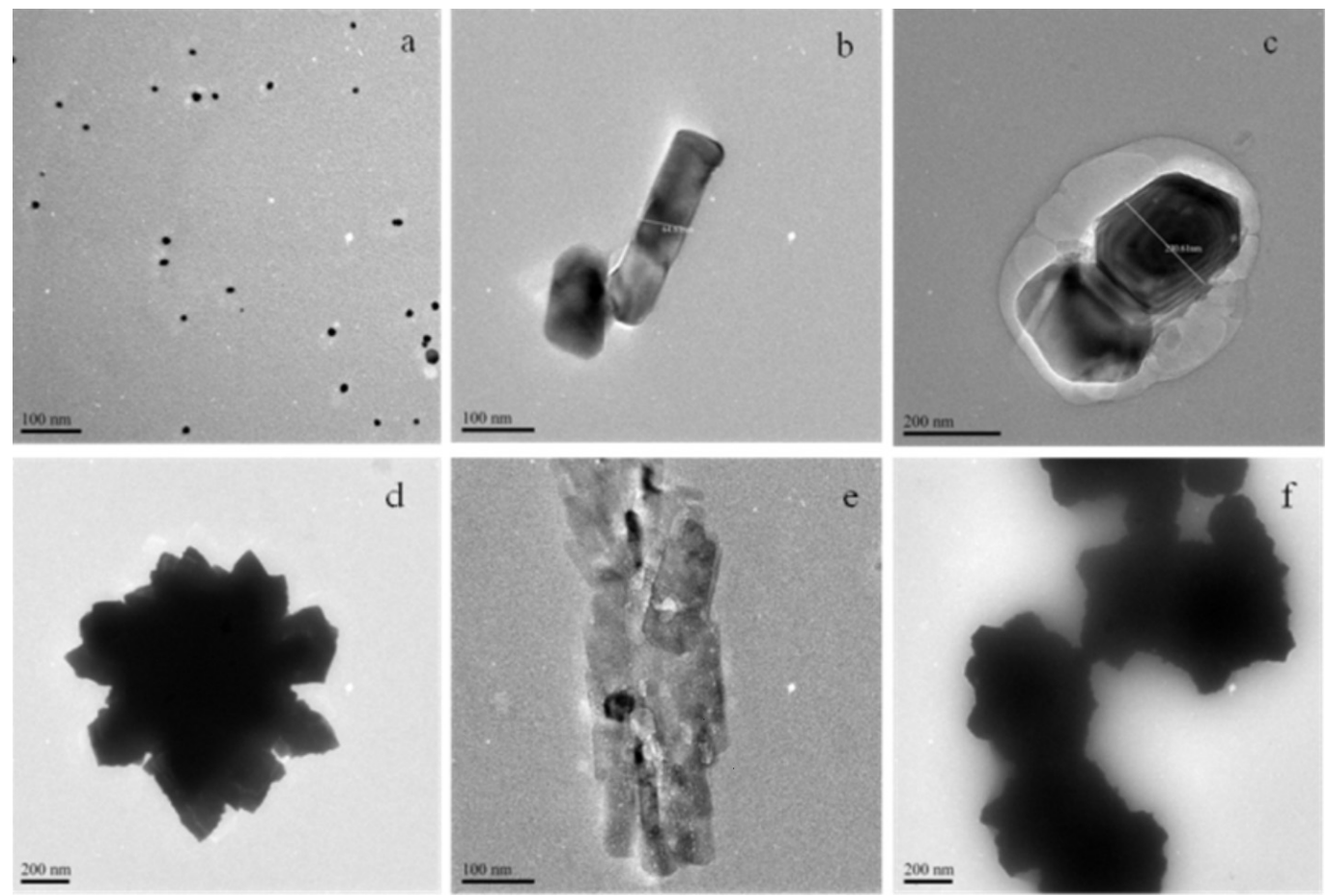

Figure 2 HRTEM of the UF-AuNPs in various DA concentrations. a-c Show growth in the size of the NPs from $0 \mathrm{M}$ (a) average diameter of $10.12 \mathrm{~nm}$, in $5 \mathrm{mM}(\mathbf{b})$ with core size of $64.61 \mathrm{~nm}$ and in $0.05 \mathrm{M}(\mathbf{c})$ with core side of $230.61 \mathrm{~nm}$, d-e show aggregates of NPs.

the AA concentrations are 200 times greater than those of DA. Above this the high concentrations of AA would interfere with the detection of DA.

\subsection{Optimization of $\mathrm{pH}$}

Dopamine is an organic base with a $\mathrm{pKa}$ of 8.9. The media $\mathrm{pH}$ can therefore affect the form of DA in aqueous solution and thus the reaction between DA and the AuNPs. Therefore, it is important to optimize the $\mathrm{pH}$ of the solution. We investigated the impact of $\mathrm{pH}$ in the range from 2 to 11 (see electronic supplementary information) and the absorbance ratio of $\mathrm{A}_{388} / \mathrm{A}_{308}$ were plotted against $\mathrm{pH}$. It must be noted that $\mathrm{DA}$ absorbs at three wavelengths $(308,388$ and $500 \mathrm{~nm})$. The $\mathrm{A}_{388} / \mathrm{A}_{308}$ ratio was chosen because most of the DA concentrations that were prepared absorb at these two wavelengths. Lower absorbance ratios $\left(\mathrm{A}_{388} / \mathrm{A}_{308}\right)$ were observed for $\mathrm{pH}<7.4$ and $\mathrm{pH}>9$. The highest absorbance ratio $\left(\mathrm{A}_{388} / \mathrm{A}_{308}\right)$ was observed at $\mathrm{pH}=8$. For $\mathrm{pH}<7.4$, the low ratio is probably due to the fact that DA is protonated

A

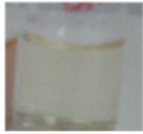

Control

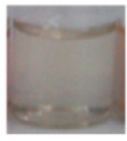

$\mathrm{UA}$

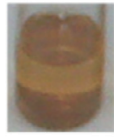

DA

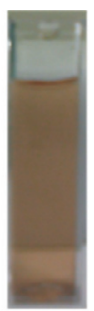

AA Mixture

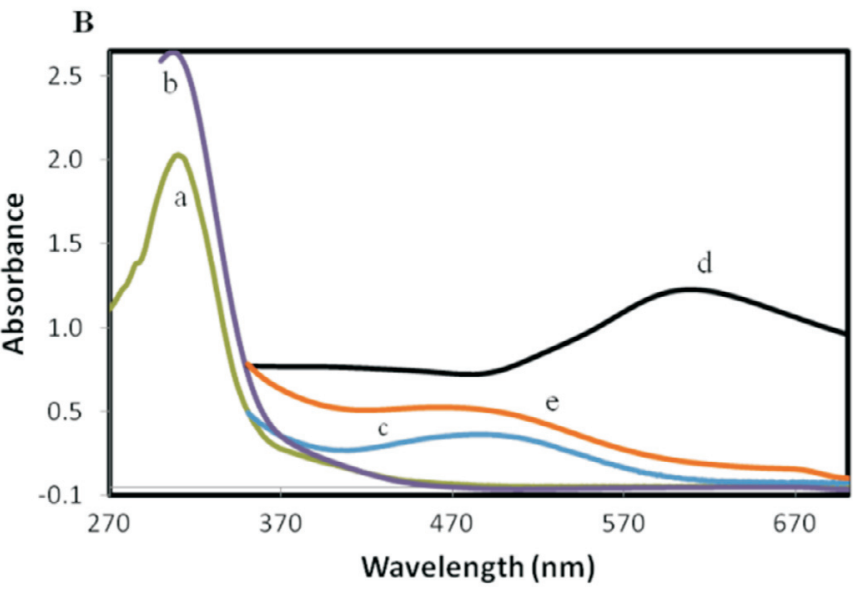

Figure 3 (A) shows the colours that resulted when the analytes reacted with the AuNPs. a, is a control, AuNPs in water; b, UA (0.125 M); c, DA (0.05 M); $\mathrm{d}, \mathrm{AA}(0.125 \mathrm{M})$ and e mixture of the three analytes; (B) depicts the absorption spectra corresponding to (A). 

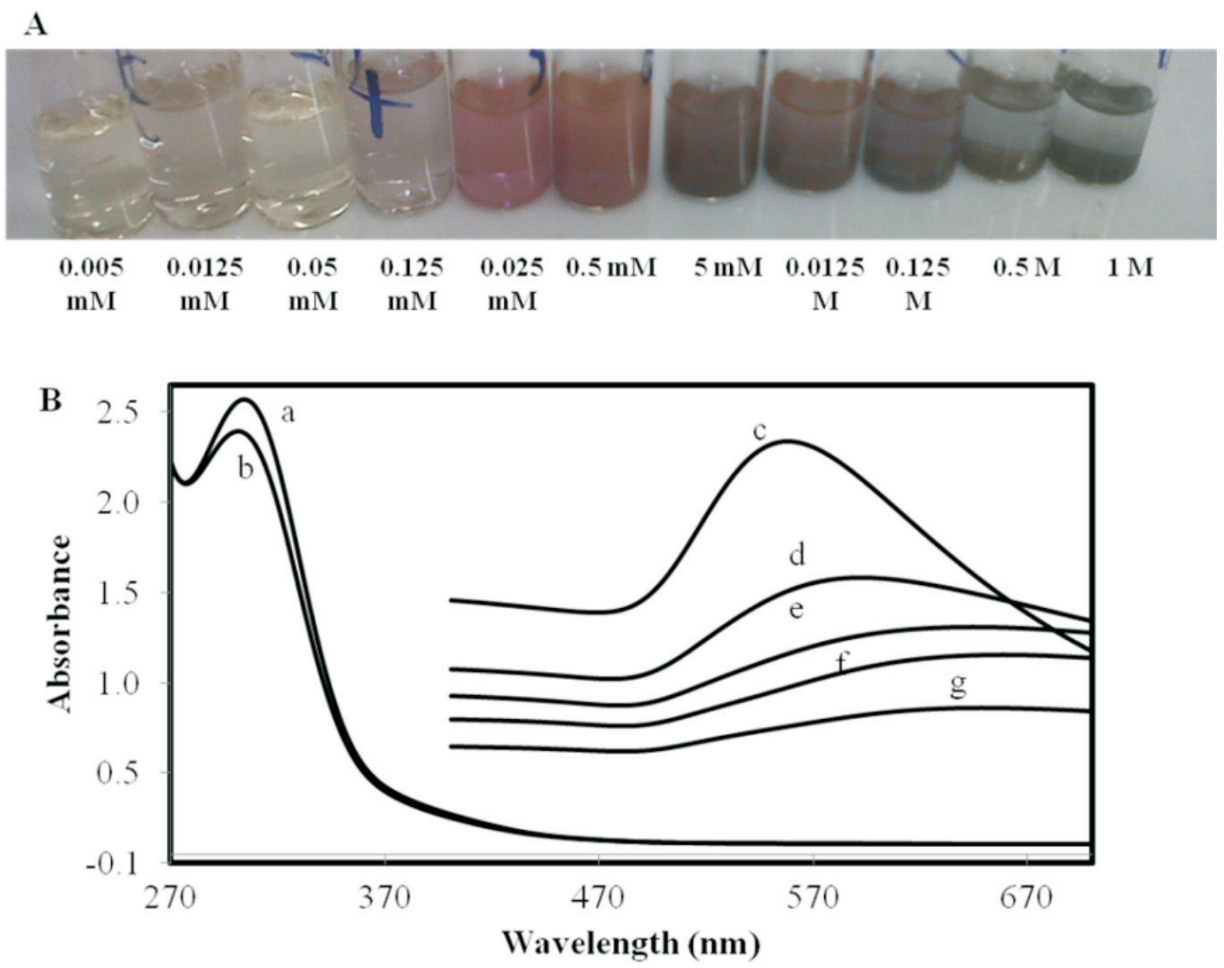

Figure 4 (A) shows the response of the probe to AA; (B) depicts the absorption spectra of the UF-AuNPs in different concentrations of AA. a, 0.005 mM; b, $0.05 \mathrm{mM}$; c, $1.25 \mathrm{mM}$; d, 2.5 mM; e, $5 \mathrm{mM}$; f, $0.0125 \mathrm{M}$; g, 0.5 M.

and relatively stable at acidic and neutral $\mathrm{pH}$; it can therefore not be used to reduce the excess $\mathrm{AuCl}_{4}{ }^{-}$or $\mathrm{AuCl}_{2}^{-}$ions. In basic media (e.g. $\mathrm{pH}$ 8), DA becomes deprotonated, ${ }^{26}$ very unstable and easily oxidized; this results in the $\mathrm{AuCl}_{4}^{-}$or $\mathrm{AuCl}_{2}^{-}$ions being easily reduced to form nanoparticles, hence resulting in a higher absorbance ratio. At $\mathrm{pH}>9$ the AuNPs were not stable and tend to aggregate rapidly, resulting in a colour change before DA is introduced (not shown). Therefore $\mathrm{pH} 8$ was chosen as the optimal media $\mathrm{pH}$.

\subsection{Detection of DA Under Optimal pH}

Under optimal $\mathrm{pH}$, colour changes were observed for solutions of DA as low as $5 \mathrm{nM}$ (Fig. 6A). The control solution $(0 \mathrm{M}$ $\mathrm{DA})$, was pale green but when DA was introduced the green colour first intensified with increasing concentration of DA ( $5 \mathrm{nM}-5 \mu \mathrm{M}$ ) and gradually changed to an orange colour (for higher concentrations of DA). As depicted in Fig. 6B, the absorbance ratio of $A_{388} / A_{308}$ gradually increased upon addition of more DA. The mechanism that brings about the colour change

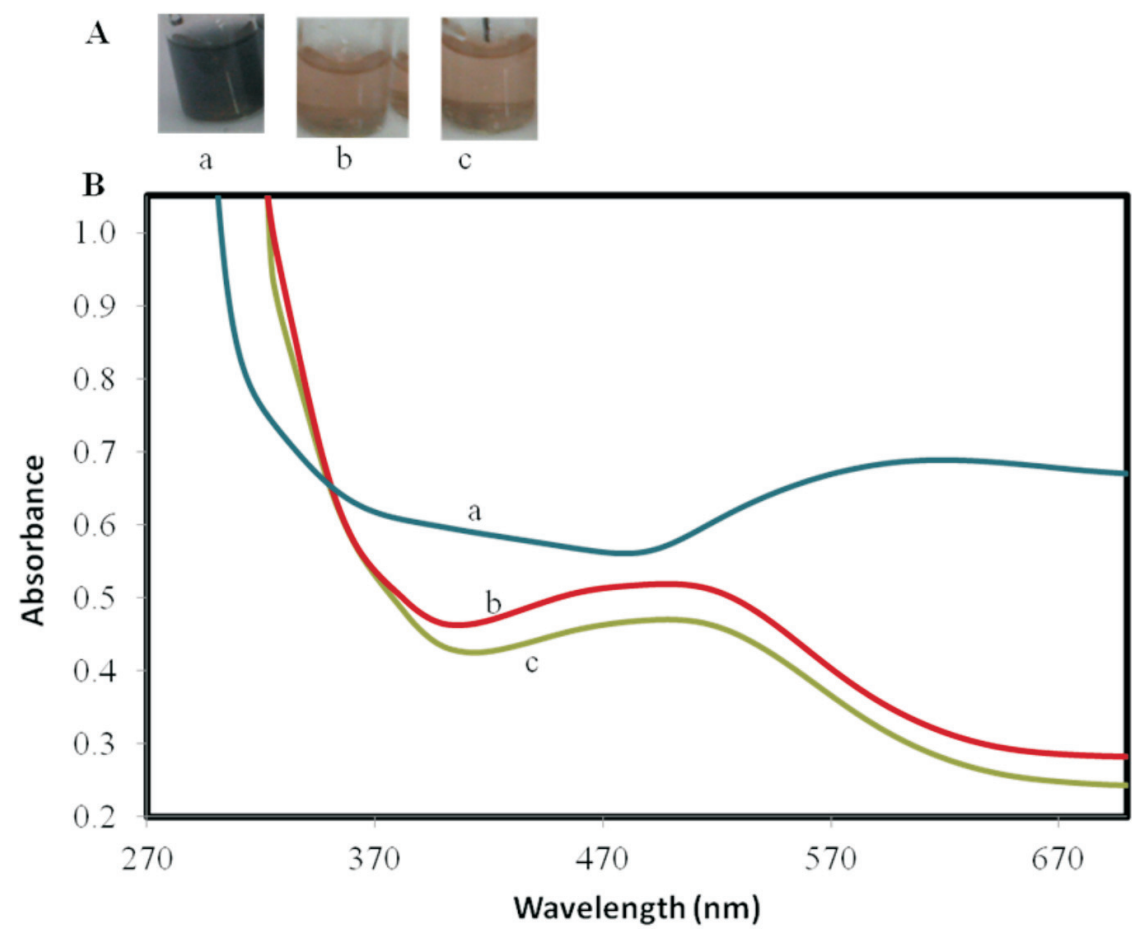

Figure 5 (A) Photograph showing the response of the probe to the mixtures of DA and AA concentrations; (B) depicts the corresponding absorption spectra of the mixtures of (a) $0.25 \mathrm{mM}: 50 \mathrm{mM}$ (DA/AA), (b) $1.25 \mathrm{mM}: 250 \mathrm{mM}$ (DA/AA), and (c) $2.5 \mathrm{M}: 0.05 \mathrm{M}$ (DA/AA). 

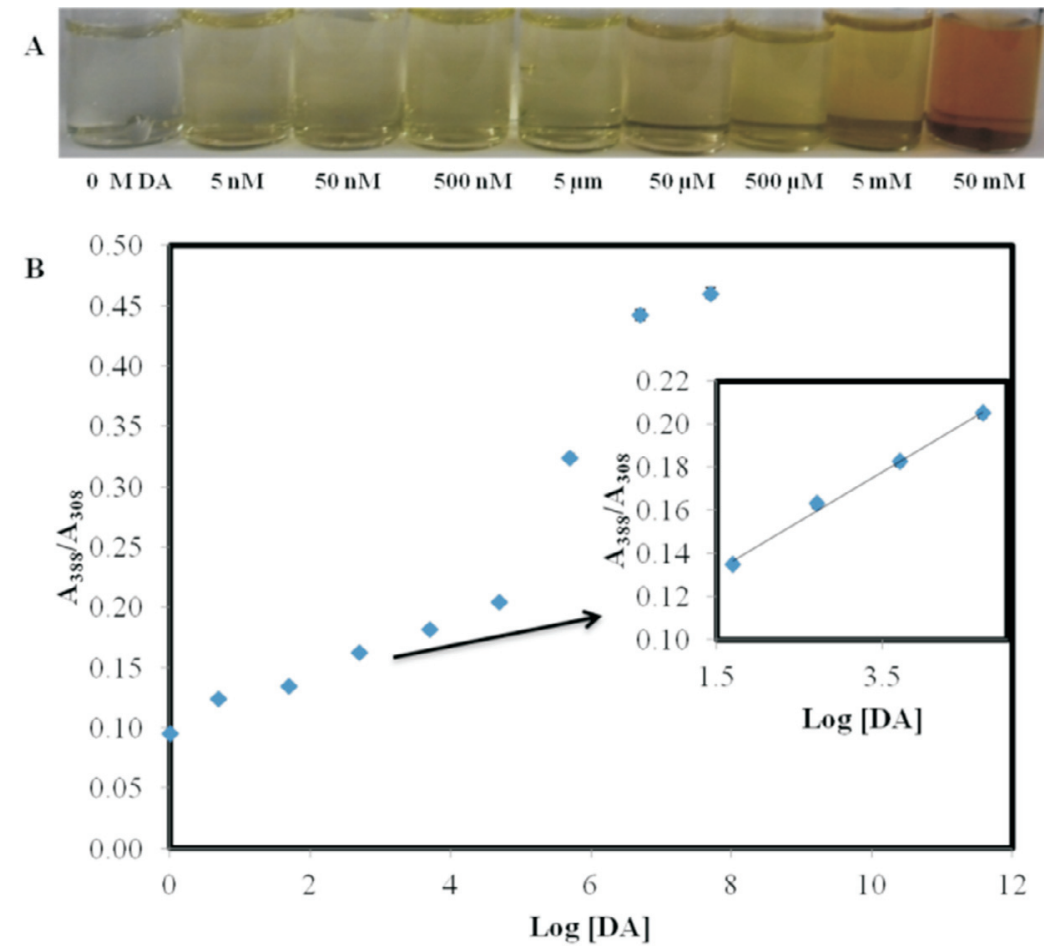

Figure 6 (A) Photograph of the UF-AuNPs in the presence of various concentrations of DA at $\mathrm{pH} 8$; (B) plots of the absorbance ratio $\left(\mathrm{A}_{388} / \mathrm{A}_{308}\right)$ versus logarithm of the concentrations of DA $(0 \mathrm{M}, 5 \mathrm{nM}, 50 \mathrm{nM}, 500 \mathrm{nM}, 5 \mu \mathrm{M}, 50 \mu \mathrm{M}, 500 \mu \mathrm{M}, 5 \mathrm{mM}$ and $5 \mathrm{mM}$, respectively); $\mathrm{n}=5$ and standard deviation $=$ 0.0047 .

is similar to the one observed previously under sub-optimal $\mathrm{pH}$ conditions, where the AuNPs were seen to become larger, changed morphology and aggregated on addition of increasingly more DA (see supporting information). Good linearity was obtained over a range of $5.0 \times 10^{-8}-5.0 \times 10^{-5} \mathrm{M}$ and the detection limit was estimated to be $2.5 \mathrm{nM}(3 \sigma)$ which is better than the limit of the recently reported probes. ${ }^{27-30}$ The method has better selectivity than other reported AuNP-based colorimetric methods for detecting DA. . $14,19,31,32^{2}$

\subsection{Detection of Dopamine in Whole Blood Sample}

Lastly, the sensitivity and selectivity of the probe towards DA in a whole blood sample was studied. The results are presented in Fig. 7. Before the addition of any AuNPs the blood sample was red in colour (Fig.7A, a). When the AuNPs were added to the solutions, a colour change from red to green was observed; the green colour intensified after spiking the solutions with DA (Fig.7A, b-d). The change of colour before spiking in Fig. 7A, b; is likely due to the presence of DA in blood. In Fig. 7B, (a) is the

\section{A}

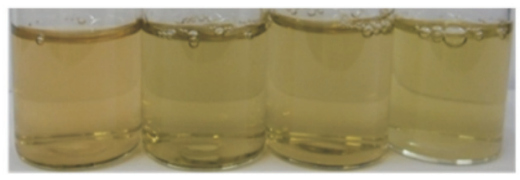
a
b c
d

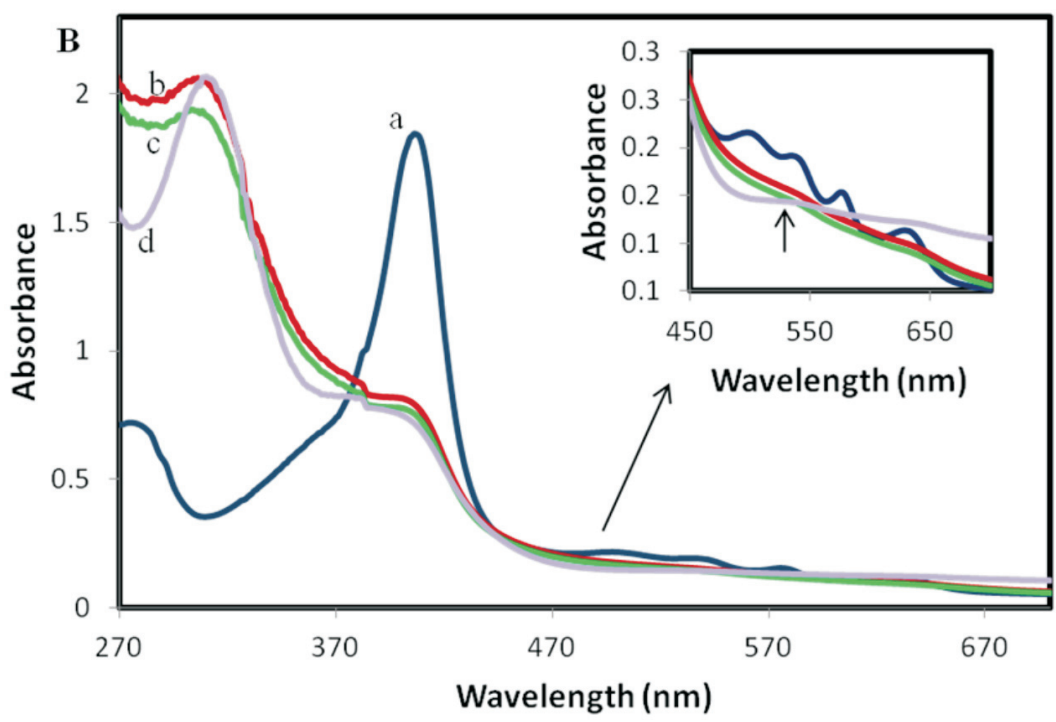

Figure 7 Detection of dopamine in real sample (whole blood). In (A), a, whole blood; b, whole blood + AuNPs; c, whole blood + AuNPs $+5 \mu$ M DA; $\mathrm{d}$, whole blood + AuNPs $+50 \mu \mathrm{M} \mathrm{DA}$; (B) depicts the absorption spectra that correspond to the solutions in (A). 
absorption spectra of the blood sample before the AuNPs were added. Apart from the main intense band at $406 \mathrm{~nm}$, several small absorption bands ( 280, 500, 538, 574 and $633 \mathrm{~nm})$ were observed, which all disappeared on addition of the AuNPs (Fig. 7B, b-c). The insert in Fig. 7B clearly shows the disappearance of the minor bands from the whole blood sample, which means that the probe was not sensitive to other interferences present in the whole blood. The additional peaks that were formed in the presence of the AuNPs around 308 and $389 \mathrm{~nm}$, shown in the spectra of b (whole blood + AuNPs), c (whole blood + AuNPs $+5 \mu \mathrm{M} \mathrm{DA}$ ) and d (whole blood + AuNPs + $50 \mu \mathrm{M}$ DA) corresponded to those that were observed for DA in the absence of whole blood. Another band was formed around $540 \mathrm{~nm}$ (Fig. 7B, d), which further confirmed the detection of DA (the increase of DA in the solution as before). The probe showed good selectivity and sensitivity for DA in a very complex matrix (whole blood), without any additional sample preparation.

\section{Conclusions}

The study developed a novel probe for detecting DA in aqueous samples by utilizing UF-AuNPs. By taking advantage of good reducing properties, DA induced growth of UF-AuNPs and the subsequent change in the morphology of the nanoparticles resulted in a clear and observable colour change from pale green to orange then brown. Spectral shifts were observed to accompany the colour changes signalling the formation of large particles or aggregates. Under non-optimized conditions, colour change was observed from $0.5 \mu \mathrm{M}$. In the mixture of DA and $\mathrm{AA}$, the probe could detect DA, even when the concentrations of AA were up to 200 times higher than those of DA. At very high concentrations of AA (more than 200 times greater than DA), a deep purple colour is observed with absorption maxima around $605 \mathrm{~nm}$, showing the interference of AA. Under optimized $\mathrm{pH}$ conditions, colour changes were observed already from $5 \mathrm{nM}$ DA and the detection limit was improved to $2.5 \mathrm{nM}$ from the previous $500 \mathrm{nM}$. The probe could selectively detect DA in whole blood sample without any sample preparation other than dilution. The practical simplicity of the probe demonstrated that it would be very useful in the development of new AuNP-based probes as it is safe and reproducible (no toxic functionalization is required) and rapid, resulting in colour changes at room temperature within $2 \mathrm{~min}$. The developed probe also gave some insight into the synthesis of various shaped AuNPs during the detection of DA, and this information is now being used to further develop sensitive probes with both free and immobilized nanoparticles.

\section{Acknowledgements}

The authors express their sincere thanks to MINTEK (Randburg, South Africa), The Henderson Postgraduate Scholarship and the NRF Scarce Skills Scholarship (South Africa) for funding. HRTEM studies we conducted at MINTEK. The experimental work was done at the Chemistry Department at Rhodes University.

\section{Supplementary Material}

Supplementary information is provided in the online supplement.

\section{${ }^{s}$ ORCID iD}

N. Khanyile: (D) orcid.org/0000-0002-9647-9006

\section{References}

1 J. Huang, Y. Liu, H. Hou and T. You, Simultaneous electrochemical determination of dopamine, uric acid and ascorbic acid using palla- dium nanoparticle-loaded carbon nanofibers modified electrode, Biosens. Bioelectron., 2008, 24, 632-637.

2 D.-S. Kim, E.-S. Kang, S. Baek, S.-S. Choo, Y.-H. Chung, D. Lee, J. Min and T.-H. Kim, Electrochemical detection of dopamine using periodic cylindrical gold nanoelectrode arrays, Sci. Rep., 2018, 8, 14049.

3 T. Ören, Ö. Birel and Ü. Anik, Electrochemical determination of dopamine using a novel perylenediimide-derivative modified carbon paste electrode, Anal. Lett., 2018, 51, 1680-1693.

4 S. Jahani and H. Beitollahi, Selective detection of dopamine in the presence of uric acid using $\mathrm{NiO}$ nanoparticles decorated on graphene nanosheets modified screen-printed electrodes, Electroanal., 2016, 28, 2022-2028.

5 Y. Haldorai, A.T.E. Vilian, M. Rethinasabapathy, Y.S. Huh and Y.-K. Han, Electrochemical determination of dopamine using a glassy carbon electrode modified with TiN-reduced graphene oxide nanocomposite, Sens. Actuators B, 2017, 247, 61-69.

6 R.M. Wightman, L.J. May and A.C. Michael, Detection of dopamine dynamics in the brain, Anal. Chem., 1988, 60, 769A-779A.

7 B. Kong, A. Zhu, Y. Luo, Y. Tian, Y. Yu and G. Shi, Sensitive and selective colorimetric visualization of cerebral dopamine based on double molecular recognition, Angew. Chem., Int. Ed., 2011, 50, 1837-1840, S1837/1831-S1837/1838

8 Y. Lin, C. Chen, C. Wang, F. Pu, J. Ren and X. Qu, Silver nanoprobe for sensitive and selective colorimetric detection of dopamine via robust Ag-catechol interaction, Chem. Commun., 2011, 47, 1181-1183.

9 E.S. Forzani, H. Zhang, W. Chen and N. Tao, Detection of heavy metal ions in drinking water using a high-resolution differential surface plasmon resonance sensor, Environ. Sci. Technol., 2005, 39, 1257-1262.

10 H. Li, J. Fan, F. Song, H. Zhu, J. Du, S. Sun and X. Peng, Fluorescent probes for $\mathrm{Pd} 2+$ detection by allylidene-hydrazone ligands with excellent selectivity and large fluorescence enhancement, Chem.: Eur. J., 2010, 16, 12349-12356.

11 X. Xu, J. Wang, F. Yang, K. Jiao and X. Yang, Label-free colorimetric detection of small molecules utilizing DNA oligonucleotides and silver nanoparticles, Small, 2009, 5, 2669-2672.

12 S. Kim, M.S. Eom, S.K. Kim, S.H. Seo and M.S. Han, A highly sensitive gold nanoparticle-based colorimetric probe for pyrophosphate using a competition assay approach, Chem. Commun., 2013, 49, 152-154.

13 Y. Song, W. Wei and X. Qu, Colorimetric biosensing using smart materials, Adv. Mater., 2011, 23, 4215-4236.

14 Y. Zheng, Y. Wang and X. Yang, Aptamer-based colorimetric biosensing of dopamine using unmodified gold nanoparticles, Sens. Actuators B, 2011, B156, 95-99.

15 Y.-R. Ma, H.-Y. Niu, X.-L. Zhang and Y.-Q. Cai, One-step synthesis of silver/dopamine nanoparticles and visual detection of melamine in raw milk, Analyst, 2011, 136, 4192-4196.

16 W. Chen, H.-H. Deng, L. Hong, Z.-Q. Wu, S. Wang, A.-L. Liu, X.-H. Lin and X.-H. Xia, Bare gold nanoparticles as facile and sensitive colorimetric probe for melamine detection, Analyst, 2012, 137, 5382-5386.

17 Y. Zhang, B. Li and X. Chen, Simple and sensitive detection of dopamine in the presence of high concentration of ascorbic acid using gold nanoparticles as colorimetric probes, Microchim. Acta, 2010, 168, $107-113$.

18 L. Liu, S. Li, L. Liu, D. Deng and N. Xia, Simple, sensitive and selective detection of dopamine using dithiobis(succinimidylpropionate)modified gold nanoparticles as colorimetric probes, Analyst, 2012, 137, 3794-3799.

19 H.-C. Lee, T.-H. Chen, W.-L. Tseng and C.-H. Lin, Novel core etching technique of gold nanoparticles for colorimetric dopamine detection, Analyst, 2012, 137, 5352-5357.

20 N. Ngomane, N. Torto, R. Krause and S. Vilakazi, A colorimetric probe for dopamine based on gold nanoparticles-electrospun nanofibre composite, Mater. Today, 2015, 2, 4060-4069.

21 X. Huang, H. Wu, X. Liao and B. Shi, One-step, size-controlled synthesis of gold nanoparticles at room temperature using plant tannin, Green Chem., 2010,12, 395-399.

22 E.K. Jeon, E. Seo, E. Lee, W. Lee, M.-K. Um and B.-S. Kim, Musselinspired green synthesis of silver nanoparticles on graphene oxide nanosheets for enhanced catalytic applications, Chem. Commun., 2013, 49, 3392-3394.

23 K.R. Brown, D.G. Walter and M.J. Natan, Seeding of colloidal Au nanoparticle solutions. 2. Improved control of particle size and shape, Chem. Mater., 2000, 12, 306-313.

24 R. Baron, M. Zayats and I. Willner, Dopamine-, L-DOPA-, adrena- 
line-, and noradrenaline-induced growth of Au nanoparticles: assays for the detection of neurotransmitters, Anal. Chem., 2005, 77, 1566-1571.

$25 \mathrm{~K}$. Min, H. Gao and K. Matyjaszewski, Use of ascorbic acid as reducing agent for synthesis of well-defined polymers by ARGET ATRP, Macromolecules, 2007, 40, 1789-1791.

26 Y. Liao, Y. Wang, X. Feng, W. Wang, F. Xu and L. Zhang, Antibacterial surfaces through dopamine functionalization and silver nanoparticle immobilization, Mater. Chem. Phys., 2010, 121, 534-540.

27 D. Wen, W. Liu, A.-K. Herrmann, D. Haubold, M. Holzschuh, F Simon and A. Eychmüller, Simple and sensitive colorimetric detection of dopamine based on assembly of cyclodextrin-modified $\mathrm{Au}$ nanoparticles, Small, 2016, 12, 2439-2442.

28 E. Detsri, K. Kamhom and C. Detsri, Microwave-assisted synthesis of unmodified gold nanoparticles for colorimetric detection of dopamine, Key Eng. Mater., 2017, 730, 167-171.
29 Z. Chen, C. Zhang and C. Wang, A colorimetric assay of dopamine utilizing melamine modified gold nanoparticle probes, Anal. Methods, $2015,7,838-841$.

30 Y. Zhang, S. Qi, Z. Liu, Y. Shi, W. Yue and C. Yi, Rapid determination of dopamine in human plasma using a gold nanoparticle-based dualmode sensing system, Mater. Sci. Eng. C, 2016, 61, 207-213.

31 J.-J. Feng, H. Guo, Y.-F. Li, Y.-H. Wang, W.-Y. Chen and A.-J. Wang, Single molecular functionalized gold nanoparticles for hydrogenbonding recognition and colorimetric detection of dopamine with high sensitivity and selectivity, ACS Appl. Mater. Interfaces, 2013, 5, 1226-1231.

32 M.R.H. Nezhad, J. Tashkhourian and J. Khodaveisi, Sensitive spectrophotometric detection of dopamine, levodopa, and adrenaline using surface plasmon resonance band of silver nanoparticles, J. Iran. Chem. Soc., 2010, 7, S83-S91. 


\section{Supplementary material to:}

N. Khanyile, R. Krause, S. Vilakazi and N. Torto,

Un-functionalized Gold Nanoparticles as a Simple Colorimetric Probe for Sensitive and Selective Detection of Dopamine,

S. Afr. J. Chem., 2019, 72, 207-214. 
Electronic supplementary information

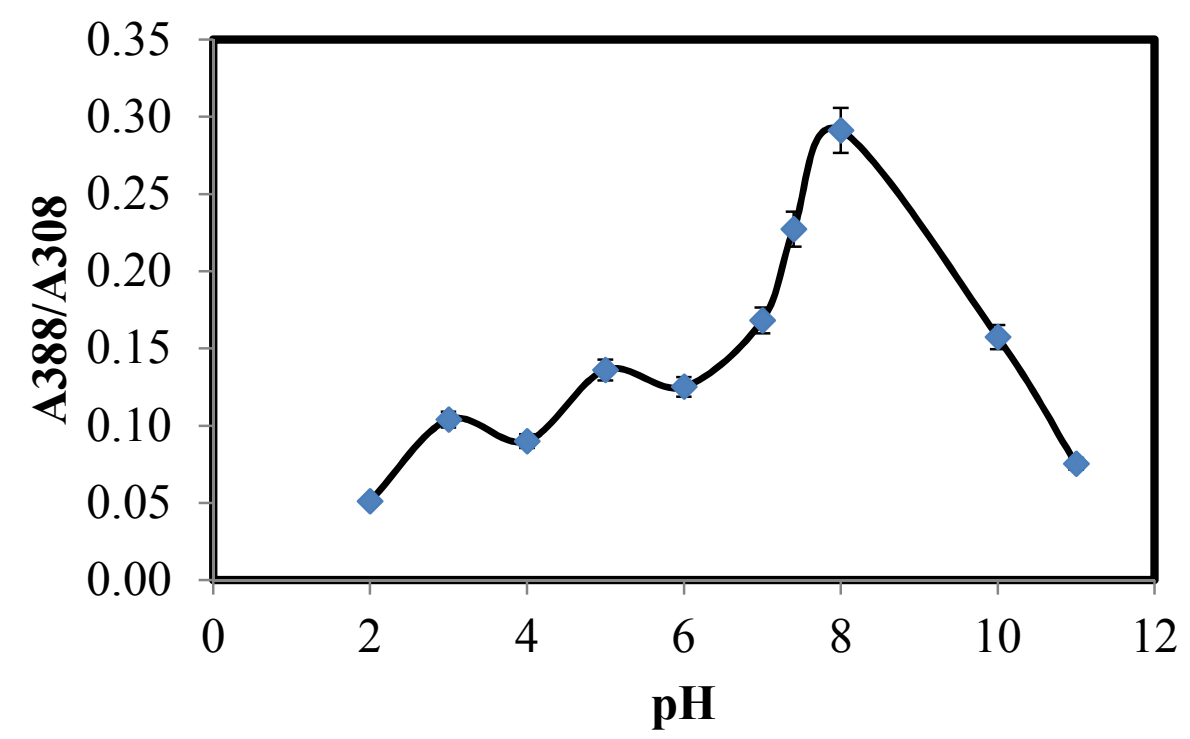

Figure 1: Effect of media $\mathrm{pH}$ on the absorbance ratio $\left(\mathrm{A}_{388} / \mathrm{A}_{308}\right)$.

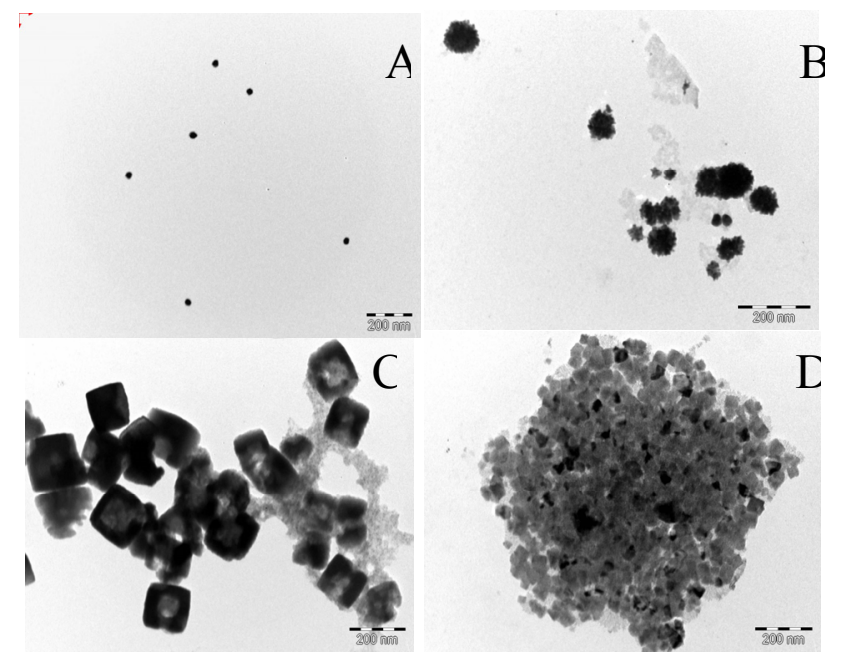

Figure 2: TEM images of the un-stabilized AuNPs in the presence of DA. A-B shows growth in the size of the nanoparticle, in $5 \mathrm{nM}$ (B) with core size of $18.37 \mathrm{~nm}$ and in $5 \mu \mathrm{M}(\mathrm{C})$ with core side of $83.66 \mathrm{~nm}, \mathrm{C}$ and D shows aggregates of nanoparticles formed in $0.05 \mathrm{mM}$ and $0.5 \mathrm{mM}$ respectively. 